\title{
INTRODUCTION
}

\section{Le parcours d'Anna Rachel Machado et son apport à l'ISD}

Jean-Paul BronCKART

(FPSE, Université de Genève)

\section{Un peu d'histoire}

C'est en septembre 1992, dans le cadre de la First Conference for Socio-cultural Research organisée à l'Universidad Complutense de Madrid, que s'est déroulée la rencontre à l'origine du vaste mouvement d'échange et de collaboration qui devait se développer plus tard entre l'interactionnisme socio-discursif (ci-après ISD) issu de Genève et les nombreux chercheurs et chercheuses du Brésil se revendiquant de cette approche. Bernard Schneuwly y avait organisé un symposium sur le thème Writing et j'avais pour ma part donné une conférence invitée intitulée Action theories, language, speech and discourse, qui présentait l'essentiel des thèmes qui allaient ensuite être affinés, organisés et publiés dans Activité langagière, textes et discours (Bronckart, 1997). A l'issue de cet exposé, un premier échange avait eu lieu avec deux chercheuses brésiliennes ayant manifesté leur intérêt pour l'approche épistémologique et méthodologique présentée ; à leurs yeux cette approche s'inscrivait idéalement dans la direction qu'elles-mêmes souhaitaient donner aux programmes de recherche en linguistique appliquée qui venaient de se mettre en place à la PUC de São Paulo. Ces deux chercheuses, que nous n'avions jamais rencontrées auparavant, étaient Maria Cecília Magalhães et Roxane Rojo ; dans la suite du colloque, Bernard Schneuwly et moi-même avons eu avec elles 
plusieurs échanges, et c'est lors du dernier d'entre eux, qui s'est tenu juste avant le "repas de gala" du 17 septembre au cours duquel nous fut confié l'organisation de la Second Conference for Socio-cultural Research (le mémorable "Vygotski-Piaget" de 1996), que nos deux collègues brésiliennes nous ont demandé s'il nous était possible de recevoir en stage à Genève une "jeune chercheuse" qui leur paraissait particulièrement prometteuse.

Nous avons bien évidemment accepté le principe de ce stage et, dans les mois qui ont suivi, les formalités administratives requises ont été efficacement réglées au Brésil et à Genève, de sorte qu'en septembre 1993 l'Unité de didactique des langues de la Section des Sciences de l'Education de la FPSE a officiellement reçu en stage une boursière doctorante dénommée Anna Rachel Machado.

Si le séjour d'Anna Rachel à Genève a duré quasiment deux années (jusqu'en juin 1995), il ne nous a fallu que quelques semaines pour appréhender et apprécier l'originalité et la vigueur d'une personnalité quasi improbable, combinant un rugueux souci de vérité et d'engagement à un inassouvible sens de la vie et de la fête. Et il nous a fallu moins de temps encore pour prendre conscience des remarquables capacités intellectuelles d'une chercheuse n'ayant absolument plus rien de la débutante.

C'est moi-même qui ai pris en charge le travail de co-orientation de la thèse de doctorat qu'Anna Rachel avait entreprise sous la direction de Maria Cecília Magalhães, thèse qui portait sur l'exploitation didactique du journal de lecture (o diário de leituras) comme genre nouveau susceptible d'enrichir les activités de réception/interprétation des textes. Les objectifs et la ligne argumentative de ce travail de thèse étaient déjà clairement établis et le corpus empirique, constitué d'une vingtaine de diários produits par des étudiants universitaires, était également recueilli et transcrit. Le problème majeur restant à traiter était dès lors, comme c'est souvent le cas, d'ordre méthodologique : d'un côté, comment saisir et conceptualiser l'ensemble des propriétés énonciatives et discursives spécifiques de chacun des textes du corpus, et d'un autre côté comment identifier dans ces mêmes textes des indices de progrès ou de développement des capacités d'interprétation textuelle. Forte de sa formation linguistique antérieure et de sa 
bonne connaissance des travaux français d'analyse de discours et de linguistique textuelle, Anna Rachel avait déjà entrepris un premier type d'analyse, consistant en l'identification et la délimitation des séquences (narrative, descriptive, explicative, etc.) telles que Adam (1990) les avait décrites et conceptualisées. Mais si cette analyse était utile et efficace (et a en conséquence constitué une part importante de l'ouvrage issu de sa thèse - cf. Machado, 1998), elle ne saisissait qu'une part des propriétés énonciatives des textes du corpus et en outre elle ne donnait aucune direction de réponse à la question des potentiels indices discursifs de développement.

\section{Quand, par un heureux hasard, les projets s'entrecroisent...}

Il se trouve qu'en cette année 1993, suite aux multiples travaux théoriques, méthodologiques et didactiques, réalisés dans l'Unité genevoise de didactique des langues, était apparue la nécessité d'élaboration d'une nouvelle synthèse qui reformulerait, réorganiserait, voire amenderait les propositions initialement formulées dans $L e$ fonctionnement des discours (Bronckart et al., 1985). Dans cette perspective, j'avais commencé à préparer les chapitres méthodologiques de la future synthèse : au plan des amendements, il s'agissait surtout de dissocier désormais le niveau des genres de textes de celui des types de discours ; au plan de la réorganisation, il s'agissait de proposer une véritable architecture textuelle qui distinguerait des niveaux superposés de structuration et identifierait les types d'opérations propres à chacun de ces niveaux.

Se manifestaient donc, à cette époque, du côté d'Anna Rachel le besoin de trouver de nouveaux modes d'analyse du corpus de textes de sa thèse, et de mon côté le besoin de conceptualiser, organiser et valider une nouvelle approche de la structuration des textes et des discours. Et la conjonction de ces deux nécessités a fait en sorte que, dans nos réunions de travail dont la fréquence s'est rapidement accrue, s'est mis en place un mouvement d'aller-retour qui enrichissait le co-orientateur tout autant que sa thésarde : les reformulations des opérations de structuration textuelle que j'élaborais pour ma part fournissaient les bases de nouvelles démarches d'analyse des textes du 
corpus de la thèse d'Anna Rachel, et l'examen, sur ce même corpus, du degré d'opérationnalité et de l'efficacité des nouveaux modes d'analyse fournissait en retour des indications susceptibles de confirmer ou d'infirmer la pertinence des propositions théoriques nouvelles. Deux faits témoignent de cet heureux croisement des parcours. Le premier est que c'est dans la thèse d'Anna Rachel publiée en 1998 que l'on trouve, pour la première fois (re-)formulés en langue lusitanobrésilienne, l'essentiel des éléments épistémologiques, théoriques et méthodologiques développés dans Activité langagière, textes et discours que j'avais publié l'année précédente. Le second est que la rédaction de la Préface de la thèse d'Anna Rachel a été effectuée en même temps - et en interaction avec- la relecture et l'établissement définitif du texte de Activité langagière. Et il faut ajouter encore et surtout que cette situation de croisement a eu comme conséquence majeure qu'Anna Rachel a disposé, d'emblée, d'une profonde maitrise théorique et pratique des propositions constitutives de l'interactionnisme sociodiscursif, maitrise qui n'a jamais cessé de se manifester dans les phases ultérieures de son parcours académique.

\section{Une œuvre trop brève, mais décisive}

Dans le cadre de cette Introduction je ne pourrai prétendre présenter de manière exhaustive, ou même simplement représentative, les travaux académiques d'Anna Rachel Machado dans leur ampleur et leur diversité, et je me bornerai en conséquence à l'évocation de quatre aspects de son œuvre qui m'ont particulièrement marqué.

Ce que je relèverai d'abord, ce sont les remarquables capacités de didactisation et/ou de vulgarisation dont notre regrettée collègue faisait preuve dans ses interventions de formation et d'enseignement. Je garde à ce propos le souvenir vivace d'une sorte de "choc admiratif" subi pourtant il y a une quinzaine d'années. En octobre 1999, après avoir donné à la PUC-SP un séminaire de $3^{\mathrm{ème}}$ cycle intitulé Développement du langage et acquisition des langues, je m'étais rendu avec divers collègues à la III Encontro Franco-Brasileiro de Analise do Discurso qui se tenait à l'Université Fédérale de Rio de Janeiro, rencontre dans le cadre de laquelle Anna Rachel donnait un minicurso consacré à la didactique des textes/discours. J'ai bien évidemment décidé de suivre 
ce cours, ce qui m'a permis d'assister à une présentation en portugais oral des genres textuels, des types de discours et des opérations langagières ; présentation qui, s'appuyant sur un ensemble de denses schémas, donnait de ces notions une image lumineuse, à la fois par sa rigueur théorique et sa limpide simplification. Ce talent didactique d'Anna Rachel n'a jamais cessé de se manifester par la suite, et je dois avouer avoir souvent exploité, dans mes interventions d'enseignement, des schémas inspirés de ceux que celle-ci avait élaborés.

Le souci pédagogique ci-dessus évoqué s'est aussi manifesté dans l'immédiate adhésion à la démarche des séquences didactiques telle que Auguste Pasquier, Joaquim Dolz et Bernard Schneuwly l'avaient conçue et développée à Genève (cf. notamment Dolz \& Schneuwly, 1998). Un des premiers projets du groupe ALTER CNPq a été en effet d'élaborer des séquences didactiques qui, tout en étant conformes aux principes généraux de ce dispositif, feraient aussi l'objet de la nécessaire adaptation aux réalités sociopolitiques et structurelles de l'enseignement du Brésil et qui pourraient également —initiative originale de ce groupe de recherche - être reconfigurées pour s'intégrer aux démarches de formation universitaire (cf. notamment Machado, 2000). Cette centration sur les séquences, dont la synthèse des résultats et des perspectives a été publiée dans A construção de modelos didáticos de gêneros (avec Vera Lúcia Lopes Cristovão, 2006), n'excluait cependant pas d'autres formes d'élaboration de matériel didactique, comme en atteste en particulier la série de manuels réalisés avec Eliane Lousada et Lília Abreu-Tardelli et publiés par les éditions Parabola de 2004 à 2007 (cf., par exemple, Machado, Lousada \& Abreu-Tardelli, 2004).

Anna Rachel Machado et son équipe de collaborateurs/trices ont cependant tôt mesuré les difficultés et problèmes de tous ordres que posait l'implémentation, en situation didactique concrète, d'un enseignement centré sur les genres, et elles ont en conséquence décidé d'ouvrir le chantier parallèle d'analyse du travail enseignant (Machado, 2003). Si cette démarche était ainsi requise par la nécessité de comprendre de quoi était fait et ce qu' exigeait l'activité enseignante réelle, elle était sous-tendue également par la volonté sociopolitique de soutenir que cette activité enseignante ne relevait ni du don ni du sacerdoce, mais constituait un véritable métier, auquel il convenait de 
se former et dont il convenait de maitriser l'ensemble des dimensions. Pour investir ce champ, Anna Rachel a pris la décision de prendre appui sur le courant de Clinique de l'activité de Clot (1995) et plus généralement sur le courant franco-français d'analyse du travail, dont fournit un exemple la contribution de Daniel Faïta à ce numéro, centrée sur les représentations discursives que des enseignants et d'autres professionnels ont de divers aspects de leur métier (cf. Article 2). Cette tentative d'articulation des principes et des propositions de l'ISD d'un côté, de l'analyse du travail à la française d'un autre, était justifiée par l'arrière-fond authentiquement vygotskien des deux démarches, même si elle impliquait quand même de tenir compte de la profonde variété des genres de textes et des types de discours, variété sur laquelle, bien que se revendiquant de Bakhtine, ce courant d'analyse du travail fait néanmoins l'impasse comme le montre encore l'étude présentée par Daniel Faïta.

Le dernier aspect du parcours de Anna Rachel Machado que je relèverais est son réinvestissement des méthodologie d'analyse issues de l'ISD ou de la linguistique textuelle, pour l'examen de la teneur des documents officiels de planification et de programmation didactiques, et pour l'étude de l'impact que pouvaient avoir ces textes sur les représentations et les pratiques des enseignants de base. Nous avions, ensemble, effectué une étude sur les procédés énonciatifs mobilisés dans les textes prescriptifs gouvernementaux émanant respectivement du Canton de Genève et du gouvernement fédéral brésilien, et mis en évidence l'impact, sur ces procédés, des considérables différences structurelles et sociopolitiques entre ces deux sortes d'Etat (Bronckart \& Machado, 2005). Avec des collègues et/ou collaboratrices, Anna Rachel avait ensuite recentré ce type d'études sur l'impact que pouvaient avoir les documents officiels et leurs injonctions, sur la construction de leur identité professionnelle (cf. Machado \& Cristovão, 2009) ou sur les dimensions affectives de leurs comportements (cf. Machado \& Abreu-Tardelli, 2009).

\section{Le plus fidèle des hommages}

Si le parcours d'Anna Rachel Machado s'est trop tôt interrompu, 
prolongement, en ce que certaines proposent des approfondissements ou enrichissements des trois axes de travail ci-dessus évoqués, et que d'autres ouvrent à de nouvelles perspectives depuis le cadre épistémologique général de l'interactionnisme socio-discursif.

Deux articles se situent dans le prolongement des travaux portant sur les genres et exploitant les séquences didactiques pour la formation des enseignants. Adair Vieira Gonçalves \& Mariolinda Rosa Romera Ferraz (Article 5) rendent compte d'une démarche consistant en l'élaboration et la gestion de séquences didactiques centrées sur la généricité, auxquelles sont étroitement associés deux enseignants de terrain ; leur analyse fait apparaître l'efficacité de cette démarche collaborative dans la maitrise des enjeux des séquences ainsi que la pertinence du travail sur les séquences pour le développement des capacités réflexives des enseignants en formation initiale. Vera Lúcia Lopes Cristovão \& Ana Paula Marques Beato-Canato (Article 4) montrent de leur côté que la centration sur les genres constitue un moyen de faire face aux besoins croissants et multiformes qui se font jour dans l'enseignement des langues, et elles proposent un schéma d'activités qui complète heureusement la méthodologie proposée dans l'ouvrage fondateur de Volochinov (1929/2010) : - consulter la littérature pertinente sur le genre concerné ; - examiner les propriétés de la communauté discursive du genre ; - rassembler un corpus d'exemplaires du genre ; - en analyser les caractéristiques contextuelles et les propriétés linguistiques internes.

Trois articles présentent des travaux relevant globalement de l'analyse du travail enseignant. La recherche présentée par Siderlene Muniz-Oliveira (Article 7) consiste en l'exploitation d'un dispositif d'instruction au sosie visant à susciter les représentations qu'ont de leur travail des professeurs d'une université publique ; l'analyse des représentations verbalisées fait apparaitre certaines des raisons du malêtre de ces enseignants, en particulier le déséquilibre, dans la réalité du travail, entre des activités requises mais désagréables (parce que bureaucratiques) et des activités didactiques innovantes pour lesquelles le temps fait défaut. Flavia Fazion \& Eliane Gouvêa Lousada (Article 9) quant à elles rendent compte des résultats obtenus par la mise en œuvre, avec une professeure de français, d'un dispositif d'autoconfrontation. Ce procédé a permis à l'enseignante de prendre conscience de certaines 
des dimensions stylistiques de son travail, notamment de ses postures corporelles, de la récurrence de certains gestes et de certains types d'action, et encore de ses modes de réaction aux événements imprévus ; et cette prise de conscience a manifestement constitué un facteur de réel développement de son autonomie professionnelle. Dans une perspective analogue, Rafaela Fetzner Drey \& Ana Maria de Mattos Guimarães (Article 8) présentent une démarche méthodologique plus diversifiée visant au développement de la professionnalité d'un enseignant en formation. Cette méthodologie articule une analyse des prescriptions émanant des superviseurs à un examen des modes de planification des stages, à des prises de données sur le déroulement effectif de l'activité d'enseignement ainsi qu'à une analyse des entretiens réalisés avec l'enseignant concerné. Effectuée dans une perspective résolument multimodale, l'analyse de cet ensemble de données fait apparaitre les conditions sous lesquelles l'étudiant en formation est susceptible de devenir véritablement acteur de cette même formation.

Luzia Bueno, Márcia Aparecida Amador Mascia \& Rafaela Scaransi (Article 3) fournissent un exemple de l'intérêt et des potentialités de l'analyse des documents officiels de prescription et/ ou de programmation. Alors que semblent officiellement adoptés au Brésil les principes d'une éducation émancipatrice visant à faire des enseignants aussi bien que des élèves, de véritables acteurs du langage, leur analyse du statut et des propriétés énonciatives des textes de Prova Brasil montre que ces derniers véhiculent en réalité une tout autre idéologie : par leur accent sur le code langagier et leur survalorisation des genres littéraires, ces textes remobilisent de fait les principes des démarches traditionnelles et les oppositions simplistes qui y sont associées.

Trois contributions nous paraissent relever de prolongements innovants eu égard aux travaux qu'avaient conduits Anna Rachel et ses collaborateurs-trices. Exploitant de manière minutieuse et efficace les apports conjoints de l'analyse du travail et de l'ISD, la recherche présentée par Ermelinda Barricelli (Article 6) a consisté à solliciter et confronter les représentations et réactions de trois personnes engagées, depuis trois angles différents, dans l'élaboration d'un document officiel d'orientation curriculaire pour l'Education infantile : celui du spécialiste de référence à fonction prescriptive, celui du formateur d'enseignants 
et celui de l'enseignant praticien représentant les destinataires du document. Outre l'intérêt de cette originale comparaison, ce travail a permis d'identifier certains indices discursifs qui s'avèrent potentiellement révélateurs de conflits latents dans l'activité et/ou sa représentation. Ecaterina Bulea Bronckart (Article 1) présente pour sa part l'approche des figures d'action qu'elle a mise en œuvre depuis quelques années, dans une version approfondie qui, à partir d'un corpus d'entretiens avec des infirmières, décrit de manière exhaustive les propriétés linguistiques de chaque figure et les diverses modalités de leur alternance dans le cours des entretiens. Cette analyse se prolonge en la défense de deux hypothèses innovantes; l'une soutenant que le potentiel développemental des figures tient à ce qu'elles sont porteuses du débat sur l'agir ayant lieu dans le milieu professionnel, débat que chaque infirmière à la fois reproduit et reconstruit de manière personnelle en cours d'entretien ; l'autre soutenant que ces figures sont des entités pleinement sémiotiques, particulièrement aptes à encadrer et exprimer les opérations psychologiques ayant trait à la praxis. Anise D’Orange Ferreira \& Glenda Cristina Valim de Melo enfin, rendent compte d'une démarche d'analyse des textes multimodaux disponibles sur le web, qui exploite d'un côté les catégories d'analyse proposées par l'ISD, d'un autre des catégories issues des approches multimodales contemporaines, notamment les clusters (ou groupes), les itinéraires thématiques parcourus dans les hypertextes et les liens de covariance entre éléments. Outre qu'elles mettent en évidence la complémentarité de ces deux ensembles de catégories d'analyse, les auteures démontrent aussi et surtout qu'il est possible et fructueux d'enrichir le cadre de l'ISD en y intégrant des aspects des conceptions multimodales qui sont compatibles avec une approche socio-interactionniste du langage ainsi qu'avec une approche des genres textuels comme instruments majeurs du développement psychologique.

La contribution de Joaquim Dolz (Article 11) enfin s'inscrit dans une perspective plus générale qui, prenant appui sur la démarche des séquences didactiques au développement et à la diffusion de laquelle Anna Rachel a largement contribué, plaide pour le renouvellement d'activités et d'exercices scolaires qui, au-delà des situations de bain de langage ou de libre expression, sont à ses yeux indispensables pour accéder à une solide maitrise langagière. Après avoir réexaminé les différentes conceptions, historiques et contemporaines, des exercices 
scolaires, il défend une approche engagée de l'ingénierie didactique qui présente deux aspects centraux. Sous l'angle des produits, concevoir les activités dans une perspective clairement vygotskienne articulant ZDP et dynamique propre des conduites des apprenants; sous l'angle du processus, élaborer ces moyens d'enseignement dans le cadre d'une démarche d'ordre scientifique, se donnant les conditions requises pour concevoir, tester et contrôler l'efficience d'activités et d'exercices scolaires à la fois riches et réellement novateurs.

\section{Mais encore...}

$\mathrm{Si}$, comme le montrent les textes que nous venons de commenter et comme d'autres textes ailleurs l'ont montré, Anna Rachel nous demeure présente et sa pensée active, elle nous manque tout autant, comme nous manquent son talent et sa gouaille.

En écho, ce petit extrait de l'œuvre de René Char, poète dont j'avais appris, un soir arrosé dans un bar de la Paulista, qu'Anna Rachel comme moi le révérait.
Puisqu'ilfaut renoncer
À ce qu'on ne peut retenir.
Qui devient autre chose
Contre ou avec le cœur, -
L'oublier rondement
Puis 6attre les 6uissons
Pour chercher sans trouver
Ce qui doit nous guérir
De nos maux inconnus
Que nous portons partout.

\section{Bibliographie}

AdAM, Jean-Michel. 1990. Eléments de linguistique textuelle. Liège: 
Bronckart, Jean-Paul. 1997. Activité langagière, textes et discours. Pour un interactionisme socio-discursif. Paris: Delachaux et Niestlé.

Bronckart, Jean-Paul, Bain, Daniel, Schneuwly, Bernard, Davaud, Clairette \& PAsquier, Auguste. 1985. Le fonctionnement des discours. Un modèle psychologique et une méthode d'analyse. Paris: Delachaux et Niestlé.

Bronckart, Jean-Paul \& Machado, Anna Rachel. 2005. En quoi et comment les textes prescriptifs prescrivent-ils? Analyse comparative de documents éducatifs brésiliens et genevois. In L. Filliettaz \& J.-P. Bronckart (éd.), L'analyse des actions et des discours en situation de travail. Concepts, méthodes et applications (pp. 221-240). Louvain: Peeters.

Clot, Yves. 1995. Le travail sans l'homme? Pour une psychologie des milieux de travail et de vie. Paris: La Découverte.

Dolz, Joaquim \& Schneuwly, Bernard. 1998. Pour un enseignement de l'oral. Initiation aux genres formels à l'école. Paris: ESF.

Machado, Anna Rachel. 1998. O diario de leituras. A introdução de um novo instrumento na escola. São Paulo: Martins Fontes.

Machado, Anna Rachel. 2000. Uma experiência de assessoria docente e de elaboração de material didático para o ensino de produção de textos na universidade. DELTA, 16, 1-25.

MACHADO, Anna Rachel. 2003. Trabalho prescrito, planificado e realizado na formação de professores: primeiro olhar. Scripta, 11, 39-53.

Machado, Anna Rachel \& CRISTOVão, Vera Lucia Lopes. 2006. A construção de modelos didáticos de gêneros: aportes e questionamentos para o ensino de gêneros. Linguagem em (Dis)curso, 6, 547-573.

Machado, Anna Rachel \& Cristovão, Vera Lucia Lopes. 2009. Representações sobre o professor e seu trabalho em proposta institucional brasileira para a formação docente. In A.-R. Machado e colaboradores, Linguagem e educação. $O$ trabalho do professor em uma nova perspectiva (pp. 117-136). Campinas: Mercado de Letras.

Machado, Anna Rachel, Lousada, Eliane \& Abreu-Tardelli, Lília. 2004. Resenha. São Paulo: Parabola.

Machado, Anna Rachel, \& Santos Abreu-Tardelli, Lília. 2009. Textos prescriptivos da educação presencial e a distância: fonte primeria do estresse do professor? In A.-R. Machado e colaboradores, Linguagem e educação. O trabalho do professor em uma nova perspectiva (pp. 101-116). Campinas: Mercado de Letras.

Volochinov, Valentin. 1929[2010]. Marxisme et philosophie du langage. Limoges: Lambert-Lucas. 